\title{
Perfil de presión arterial e historia familiar de hipertensión en niños escolares sanos de Santiago de Chile
}

\author{
Marlene Aglony $\mathrm{I}^{1}$, Pilar Arnaiz $\mathrm{G}^{2}$, Mónica Acevedo $\mathrm{B}^{3}$, \\ Salesa Barja $Y^{4}$, Sonia Márquez $U^{1}$, Beatriz G uzmán $A^{5 a}$, \\ Ximena Berríos $\mathrm{C}^{5}$. \\ Blood pressure and family history \\ of hypertension in children \\ from Santiago, Chile
}

Background: The prevalence of pediatric arterial hypertension (AHT) is approximately $1 \%$ to $2 \%$. In the last ten years, mean blood pressure levels (BP) have raised due to obesity and changes in lifestyles. Family history (FH) of AHT is a risk factor to develop AHT in children. Aim: To assess blood pressure, cardiovascular risk factors and family history in healthy children of Santiago. Material and methods: Blood pressure, family history of AHT, birth weight (BW), gestational age, puberal stage, blood glucose, serum lipids and ultrasensitive Reactive C Protein (usCRP) were analyzed, using data from a study of early markers of atherosclerosis in children. Results: Data of 112 children aged between 6-12 years was analyzed. Hypertension (BP >percentile 95) was detected in 2.7\% and pre hypertension (BP in percentiles 90-95) in 3.6\% of the sample. Children with abnormal BP had higher levels of usCRP $(\mathrm{p}<0.05)$ and a non significant tendency towards a higher body mass index. All hypertensive and one pre hypertensive children had FH of AHT. Eleven percent of parents, had high blood pressure. In no children, both parents were hypertensive. Children with a family history of hypertension had higher concentrations of total serum cholesterol ( $\mathrm{p} \varangle 0.05$ ). Conclusions: The abnormal prevalence of AHT found in this study is comparable to other studies. FH associated to higher levels of BP in children. Children with abnormal BP had a higher subclinical level of inflammation (Rev Méd Chile 2009; 137: 39-45).

(Key w ords: C-reactive protein; Child; Hypertension; Infant)

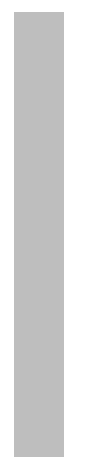

Recibido el 31 de enero, 2008. Aprobado el 1 de octubre, 2008.

Financiamiento: Estudio de investigación financiado por el Centro de Investigaciones Médicas de la Pontificia Universidad Católica de Chile (Concurso de Investigación para Becados año 2006) y por el Departamento de Pediatría de la Pontificia Universidad Católica de Chile (Concurso de Investigación para Académicos 2006).

Este estudio recibió premio al «Mejor Trabajo Clínico»del Congreso Conjunto de las Sociedades Chilenas de Nefrología, Hipertensión y Trasplante. Pucón, Septiembre de 2007.

${ }^{1}$ Unidad de Nefrología y ${ }^{2}$ Unidad de Cardiología, Departamento de Pediatría, Pontificia Universidad Católica de Chile. ${ }^{3}$ Departamento de Enfermedades Cardiovasculares, Pontificia Universidad Católica de Chile. ${ }^{4}$ Unidad de Nutrición, Departamento de Pediatría, Pontificia Universidad Católica de Chile. ${ }^{5}$ Departamento de Salud Pública, Escuela de Medicina, Pontificia Universidad Católica de Chile.

aEnfermera Universitaria

Correspondencia a: Dra. Marlene Aglony Imbarack. Unidad de Nefrología, Departamento de Pediatría, Escuela de Medicina, Pontificia Universidad Católica de Chile. Fax: 56-2-6384307.

E mail: maglony@med.puc.cl 
$\mathrm{E}^{\mathrm{n}}$ adultos la hipertensión arterial (HTA) es el factor de riesgo cardiovascular más prevalente, afectando a más de un billón de personas en el mundo. Está implicada en más de 7 millones de muertes anuales, siendo una de los principales contribuyentes a la enfermedad cardiovascular y accidente cerebrovascular ${ }^{1}$, dado lo cual se han desarrollado importantes programas de prevención y estudios en la edad pediátrica ${ }^{2}$.

La HTA en pediatría es una patología subdiagnosticada y por lo general asintomática, describiéndose en la literatura una prevalencia de $1 \%$ a $2 \%{ }^{3}$. Estudios en escolares reportan prevalencias de hasta 4,5\%4.

En los últimos años se ha producido un vuelco epidemiológico en la población infantil cobrando gran protagonismo la obesidad. Existe una fuerte asociación entre obesidad e HTA. Sorof y cols en múltiples publicaciones sobre el tema ${ }^{4,5}$ y después de estudiar más de 2.460 estudiantes entre 12 y 16 años, encontró una prevalencia de hasta 34\% de HTA en niños con índice de masa corporal mayor a percentil $95^{5}$. En Chile se ha modificado negativamente el estilo de vida de la población, con un aumento de todos los factores de riesgo tradicionales, tanto en la población adulta como en niños y adolescentes. Se teme que la obesidad, el sedentarismo y el síndrome metabólico serán altamente prevalentes en este milenio, con un aumento en el riesgo cardiovascular desde la edad pediátrica y con el desamollo de eventos comonarios prematuros en la edad adulta ${ }^{6-11}$. Estadísticas de la Junta Nacional de Auxilio Escolar y Becas (JUNAEB) de 2006 (considerando obesidad a un índice de masa corporal sobre percentil 95) muestran un promedio de 19,4\% de niños obesos en el primer año básico de todas las escuelas del país ${ }^{12}$. La obesidad es un factor de riesgo cardiovascular modificable, presente desde la niñez y que se mantiene en alto porcentaje en la edad adulta, aumentando el niesgo de enfermedad aterosclerótica $^{13}$. En un estudio pionero a nivel nacional, recientemente publicado por el grupo de Cardiología Preventiva de nuestra institución, se evidenció una mayor carga de factores de riesgo clásicos y un estado proinflamatorio asociado a la génesis de la ateroesclerosis en niños chilenos obesos al ser comparados con sus pares eutróicos ${ }^{14}$.

La historia familiar de HTA también es un factor de riesgo importante para desarrollar hipertensión. Aunque sus niveles de presión arterial se encuentran bajo el rango hipertensivo, los hijos de padres hipertensos tienden a presentar mayores cifras tensionales que los hijos de normotensos ${ }^{15}$. Se ha descrito un aumento del riesgo de HTA de hasta $20 \%$ en el caso de que uno de los padres sea hipertenso y de hasta $50 \%$ si ambos lo son ${ }^{16}$. En relación a la presión arterial de los niños y el posterior desarrollo de HTA primaria, estudios familiares longitudinales han permitido establecer una interrelación entre la carga genética y el medioambiente $^{17}$. Se han descrito mutaciones y polimorfismos de genes involucrados en el control de la presión arterial, como es el caso del receptor de la enzima convertidora de angiotensina, canal epitelial de sodio amiloride sensible, etc ${ }^{18}$.

El objetivo de este estudio es describir la presión arterial de una población de niños sanos de Santiago y su asociación a otros factores de riesgo cardiovascular y antecedente familiar de HTA.

\section{Pacientes y MÉTOdo}

Estudio de cohorte realizado entre octubre de 2005 y agosto de 2006 en Santiago, en niños procedentes de estratos socioeconómicos medio, medio-bajo y bajo. Se estudiaron hijos de participantes de un estudio epidemiológico previo, realizado por el Departamento de Salud Pública de la Pontificia Universidad Católica de Chile. Los criterios de inclusión fueron: edad entre 6 y 13 años, sin historia personal de trastomos del metabolismo de la glucosa, dislipidemia, HTA ni enfermedades metabólicas y ausencia de enfermedades infecciosas, terapia farmacológica recientes. De los datos obtenidos en el estudio, se analizaron: antecedente familiar de HTA, peso de nacimiento (PN) y edad gestacional (EG), encuesta sobre factores de riesgo cardiovascular, antropometría, presión arterial, evaluación puberal, glicemia, perfil lipídico y proteína $\mathrm{C}$ reactiva ultrasensible (PCRus) en ayunas.

Se les solicitó acudir en ayuno de $12 \mathrm{~h}$ e ingesta de alimentos livianos (previamente indicados) por $24 \mathrm{~h}$ previas a su evaluación. Una enfermera realizó a cada niño una encuesta general que incluía antecedentes de peso de nacimiento, edad gestacional (considerándose prematurez una edad gestacional menor a 38 semanas al momento del parto), antecedentes familiares de HTA y las mediciones antropométricas y toma de presión arterial. El examen físico fue hecho por una pediatra cardióloga. Luego se 
realizó la extracción de muestras sanguíneas bajo condiciones estandarizadas.

Mediciones antropométricas y evaluación puberal. En cada niño se midió peso y talla en forma controlada, con balanza de palanca y estatímetro marca SECA ${ }^{\circledR}$, descalzos, vestidos con ropa interior y una bata. Se calculó el índice de masa corporal (IMC: Peso $(\mathrm{Kg}) /$ Talla $\left.(\mathrm{m})^{2}\right)$, expresado en percentiles y como puntaje $\mathrm{z}$ ( $\mathrm{z}=$ =valor medido - mediana/1DS). Se definió obesidad como índice de masa corporal (IMC) igual o mayor al percentil 95 para edad y sexo; y eutrofia entre percentil 10 y 84 . Se utilizaron como referencia las curvas NCHS-CDC año $2000^{19}$ y las normas para evaluación nutricional del niño del Ministerio de Salud ${ }^{20}$. Para estimar obesidad central se midió el perímetro de cintura con cinta métrica inextensible, sobre el borde lateral superior de la cresta ilíaca derecha, al final de una espiración normal ${ }^{19}$, promediándose dos mediciones. Se utilizó como referencia una distribución percentilar en población infantil multiracial, expresándose como porcentaje del pc 50 (Pcp 50) =(valor medido/pc 50) $\mathrm{x} 100^{21}$. Por último se midieron cuatro pliegues cutáneos: bicipital, tricipital (TC), subescapular (SE) y suprailíaco con un "cáliper" Lange ${ }^{\circledR}$ y circunferencia braquial de acuerdo a normas internacionales ${ }^{22}$. Se usó la razón de SE/TC como indicador indirecto de grasa troncal y se estimó el porcentaje de masa grasa total a través de las fórmulas de Slaughter ${ }^{23}$. El estadio de maduración sexual se evaluó mediante criterios de Tanner ${ }^{20}$.

Medición de presión arterial. La presión arterial se tomó en tres controles cada $5 \mathrm{~min}$, en decúbito supino, después de 10 min de reposo y en el brazo derecho, promediándose los valores. Se utilizó un equipo oscilométrico automático (Dynamap Pro 100, Criticon) con manguito adecuado para el tamaño del brazo del niño (mango pediátrico escolar y de adulto). Según la última clasificación de la Task Force sobre diagnóstico y manejo de HTA en niños y adolescentes se considera ${ }^{24}$ : presión arterial normal (normotensión) si la presión arterial sistólica (PAS) 0 diastólica (PAD) es menor del percentil 90; HTA si la PAS o la PAD es mayor o igual al percentil 95 para sexo, edad y talla y estado prehipertensivo en los casos que la PAS o PAD se encuentra entre el percentil 90 y percentil 95 para sexo, edad y talla. Los niños hipertensos se subdividieron en 2 catego- nías: HTA estadio 1 cuando la PAS o PAD se encuentra entre el percentil 95 y percentil 99 más 5 mmHg y en HTA estadio 2 cuando la PAS o PAD es mayor a percentil 99 más $5 \mathrm{mmHg}$. Para efectos de comparación se estableció el porcentaje de diferencia de las PAS y PAD de cada paciente con respecto a su percentil 50 (sexo, edad y talla), los valores (+) se encuentran sobre el percentil 50 correspondiente y los (-) bajo éste.

Exámenes de sangre. La proteína C-reactiva ultrasensible fue medida por método nefelométrico Dade Behring (Nefelómetro Dade Behring BN II) con límite de detección 0,1 mg/L La glicemia por método de glucosa oxidasa y el colesterol total, CHDL y triglicéridos fueron medidos usando los métodos enzimáticos estándar (analizador Yací). Se calculó CLDL mediante la fórmula de Friedwald ${ }^{25}$.

Ética. El adulto responsable firmó un consentimiento informado para la participación de cada niño en el estudio, el que fue previamente aprobado por el Comité de Ética de la Escuela de Medicina de la Pontificia Universidad Católica de Chile.

Estadística. Para la descripción de resultados, usamos promedio y desviación estándar (DE) para describir las variables continuas. Se consideró significativo una $\mathrm{p}<0,05$. Dado que la PCRus no se distribuyó en forma normal, para los análisis se usó su forma logarítmica.

\section{RESULTADOS}

Se estudiaron 112 niños, edad promedio de 10,6 años, 58 de ellos hombres (52\%).

Se pesquisaron 7 niños con presión arterial en rango anormal (6,3\%): 3 niños con presiones arteriales en rango de HTA $(2,7 \%)$ y 4 con preHTA $(3,6 \%)$. Ambas categorías estuvieron dadas por cifras de presión arterial sistólica elevada, ya que ningún niño tuvo PAD anormal. Todos los niños hipertensos se encontraban en estadio 1 (Tabla 1). De los 7 niños con cifras tensionales anormales, 2 eran varones y pertenecían al grupo de preHTA. La edad promedio del grupo con PA anormal fue de 9,8 años, en el grupo prehipertensivo de 9,5 años y la de los niños hipertensos de 10,1 años. Ninguno de ellos tenía antecedente de prematurez ni de 
Tabla 1. Distribución de las presiones arteriales en el grupo de niños estudiados

\begin{tabular}{|lcc|}
\hline & $\begin{array}{c}\text { Presión arterial sistólica } \\
\mathbf{n}(\%)\end{array}$ & $\begin{array}{c}\text { Presión arterial diastólica } \\
\mathbf{n}(\%)\end{array}$ \\
\hline Normal & $105(93,7)$ & $112(100)$ \\
Prehipertensión & $4(3,6)$ & 0 \\
HTA estadio 1 & $3(2,7)$ & 0 \\
HTA estadio 2 & $0 \quad 0$ \\
Total & $112(100)$ & $112(100)$ \\
\hline
\end{tabular}

peso de nacimiento menor de 3.000 g. En relación al diagnóstico nutricional, sólo uno era obeso y éste se encontraba en categoría de prehipertensión.

Al comparar los niños de los distintos grupos, quienes tenían presión arterial anormal presentaron un nivel significativamente mayor de PCRus que el grupo de niños normotensos ( $3,02 \pm 5,0 \mathrm{v} / \mathrm{s} 0,89 \pm 1,5$ $\mathrm{mg} / \mathrm{L})$ ( $\mathrm{p}<0,05)$. Al comparar ambos grupos, los con PA anormal y normal, según el porcentaje en que sus cifras tensionales se alejaban del percentil 50, el grupo con presión arterial anormal, como era esperable tenía valores francamente más elevados, tanto en la PA sistólica $(+16 \pm 4,0 \mathrm{v} / \mathrm{s}-2,4 \pm 6,0 \mathrm{~mm}$ de $\mathrm{Hg})$ como en la PA diastólica $(+3,8 \pm 12,5 \mathrm{v} / \mathrm{s}-9,7 \pm 8,6 \mathrm{~mm}$ de $\mathrm{Hg})(\mathrm{p} \varangle 0,05)$. Sin embargo las cifras diastólicas no alcanzaron límites de anormalidad. No se encontraron diferencias estadísticamente significativas en el análisis de estos grupos en relación a edad, sexo, peso de nacimiento, edad gestacional, grado de obesidad, perfil lipídico y glicemia (Tablas 2 y 3).

Todos los niños hipertensos y uno de los prehipertensos tenían antecedente familiar de HTA. En la población estudiada se pesquisaron 25 de 224 padres con antecedente de HTA (11,2\%), 14 de ellos hombres (56\%). Ninguno de los niños tenía ambos padres hipertensos. Los niños con historia familiar positiva de HTA presentan significativamente mayores niveles de PAS $(+2,5 \pm 9,2 \mathrm{v} / \mathrm{s}-2,3 \pm 6,5)$, de PAD $(-4,0 \pm 12 \mathrm{v} / \mathrm{s}$ $-10,3 \pm 8,1)$ y de colesterol total $(161,0 \pm 16,5 \mathrm{v} / \mathrm{s} 145,0$ $\pm 25,0)(\mathrm{p} \varangle 0,05)$ que aquellos sin historia familiar. En el resto de las variables estudiadas: sexo, edad, peso de nacimiento, edad gestacional, grado de obesidad,

\section{Tabla 2. Parámetros de historia y examen físico analizados en el grupo poblacional total y análisis de subcategorías según cifras de presiones arteriales y antecedente familiar de hipertensión arterial}

\begin{tabular}{|c|c|c|c|c|c|}
\hline Variable & $\begin{array}{l}\text { Población total } \\
\text { ( } \mathrm{n}=112)\end{array}$ & $\begin{array}{l}\text { Normotensos } \\
(\mathrm{n}=105)\end{array}$ & $\begin{array}{l}\text { PA anormal } \\
(\mathrm{n}=7)\end{array}$ & $\begin{array}{l}\text { HF (-) } \\
(n=87)\end{array}$ & $\begin{array}{l}H F(t) \\
(n=25)\end{array}$ \\
\hline Sexo masculino & $52 \%$ & $53 \%$ & $28,6 \%$ & $56 \%$ & $36 \%$ \\
\hline Edad (años) \pm DS & $10,6 \pm 1,9$ & $10,6 \pm 1,8$ & $9,8 \pm 2,6$ & $10,6 \pm 1,9$ & $10,3 \pm 1,8$ \\
\hline$P N(g) \pm D S$ & $3.316 \pm 601$ & $3.363 \pm 615$ & $3.576 \pm 356$ & $3.288 \pm 631$ & $3.147 \pm 486$ \\
\hline $\mathrm{EG}(\mathrm{sem}) \pm \mathrm{DS}$ & $40 \pm 6,0$ & $40 \pm 6,2$ & $39 \pm 2$ & $39 \pm 6,6$ & $36 \pm 2,2$ \\
\hline $\mathrm{zIMC} \pm \mathrm{DS}$ & $0,5 \pm 1,0$ & $0,48 \pm 1,0$ & $0,63 \pm 0,97$ & $0,45 \pm 0,98$ & $0,66 \pm 0,9$ \\
\hline Obesidad (\% IMC >Pc95) & 13,4 & 11 & 14 & 9,8 & 12 \\
\hline $\mathrm{PAS}(\%) \pm \mathrm{DS}^{\wedge}$ & $-1,2 \pm 7,4$ & $-2,4 \pm 6,0^{*}$ & $+16 \pm 4,0^{*}$ & $-2,3 \pm 6,5^{\#}$ & $+2,5 \pm 9,2^{\#}$ \\
\hline $\mathrm{PAD}(\%) \pm \mathrm{DS}^{\wedge}$ & $-8,9 \pm 9,4$ & $-9,7 \pm 8,6^{*}$ & $+3,8 \pm 12,5^{*}$ & $-10,3 \pm 8,1^{\#}$ & $-4,0 \pm 12^{\#}$ \\
\hline
\end{tabular}

Para efectos de análisis se estableció el porcentaje de diferencia de las PASy PAD de cada paciente con respecto a su pc 50 (sexo, edad y talla), los valores (+) se encuentran sobre el pc 50 comespondiente y los (-) bajo éste. $*_{p}<0,05 ;$; $<<0,05$

$\mathrm{PN}=$ Peso nacimiento, $\mathrm{EG}=$ Edad gestacional, IMC =Indice de masa corporal, PAS =Presión arterial sistólica, PAD =Presión arterial diastólica, $\mathrm{HF}=$ Historia familiar. 
Tabla 3. Parámetros de laboratorio analizados en el grupo poblacional total y análisis de subcategorías según cifras de presiones arteriales y antecedente familiar de hipertensión arterial

\begin{tabular}{|llllll|}
\hline Variable & $\begin{array}{l}\text { Población total } \\
\text { (n=112) }\end{array}$ & $\begin{array}{l}\text { Normotensos } \\
\text { (n=105) }\end{array}$ & $\begin{array}{l}\text { PA anormal } \\
\text { (n=7) }\end{array}$ & $\begin{array}{l}\text { H F (-) } \\
\text { (n=87) }\end{array}$ & $\begin{array}{l}\text { H F (+) } \\
\text { (n=25) }\end{array}$ \\
\hline Colesterol total (mg/dl) \pm DS & $149 \pm 24,4$ & $148,8 \pm 24,5$ & $156,0 \pm 22,0$ & $145,0 \pm 25,0^{\#}$ & $161,0 \pm 16,5^{\#}$ \\
Colesterol HDL (mg/dl) \pm DS & $56,0 \pm 11,2$ & $56,2 \pm 11,5$ & $56,0 \pm 5,0$ & $55,9 \pm 11,0$ & $57,0 \pm 11,9$ \\
Colesterol LDL (mg/dl) \pm DS & $79,5 \pm 21,5$ & $79,4 \pm 21,1$ & $82,0 \pm 25,0$ & $76,8 \pm 22,0$ & $88,9 \pm 16,4$ \\
Triglicéridos (mg/dl) \pm DS & $66,0 \pm 32,2$ & $65,9 \pm 30,8$ & $71,0 \pm 49,0$ & $63,4 \pm 32,0$ & $76,0 \pm 31,5$ \\
PCRus \pm DS & $1,03 \pm 1,95$ & $0,89 \pm 1,5^{*}$ & $3,02 \pm 5,0^{*}$ & $0,83 \pm 1,4$ & $1,7 \pm 3,2$ \\
Glicemia (g/dl) \pm DS & $84,0 \pm 5,4$ & $84,4 \pm 5,5$ & $85,0 \pm 5,0$ & $84,5 \pm 5,6$ & $84,0 \pm 4,9$ \\
\hline
\end{tabular}

${ }^{*} \mathrm{p}<0,05 ;{ }^{*} \mathrm{p}<0,05$

PCRus =Proteína $\mathrm{C}$ reactiva ultrasensible, $\mathrm{HF}=$ Historia familiar

colesterol HDL, colesterol LDL, triglicénidos, PCRus y glicemia, no se encontraron diferencias con significancia estadística $(p>0,05)$.

\section{DisCUSIÓN}

En este estudio se demostró que el antecedente de HTA en la familia se asocia a mayores cifras tensionales en los hijos y que los niños con PA anormal, ya sea con hipertensión o prehipertensión, tienen un mayor nivel de inflamación subclínica, medido por PCRus.

En esta muestra pequeña de niños de Santiago, se encontró una prevalencia de HTA de 2,7\%, lo que coincide con lo reportado en la literatura ${ }^{3,4}$. Sorof y $\operatorname{cols}^{26}$ realizaron una evaluación inicial a niños sanos en edad escolar (58\% hispanos y $32 \%$ afroamericanos), y encontraron que de los escolares que resultaron hipertensos $97 \%$ correspondía a HTA sistólica. Flynn ${ }^{27}$, a través de monitorización ambulatoria continua de presión arterial, describe que en los casos de HTA secundaria, las cifras de PA diastólicas son significativas mayores durante el día y en la noche. Todos estos datos hacen pensar que la prevalencia de HTA sistólica encontrada en nuestro estudio es consistente con estudios previos, al igual que el patrón de presión arterial de tipo netamente sistólico correspondería a HTA primaria.

Se ha reportado que uno de los factores más importantes a la hora de medir la prevalencia de hipertensión arterial en la población pediátrica es la adecuada metodología en la toma de la presión arterial. Así, se ha descrito en estudios epidemio- lógicos en escolares una prevalencia de 8,7\% en la toma inicial de presión arterial, que llega a disminuir a valores cercanos a $2 \%$ en la tercera toma ${ }^{28}$. La fortaleza de nuestros resultados se basa en este concepto, ya que la toma de presión arterial fue seriada y se realizó según las recomendaciones internacionales ${ }^{24}$, y bajo condiciones estandarizadas y reproducibles (manguito y ambiente adecuado, etc), que posteriormente fueron promediadas $\mathrm{y}$, no sólo en una toma de presión aislada, como ha sido descrita en otros estudios.

El haber pesquisado 3,6\% en estado prehipertensivo de la población en edad pediátrica en nuestro estudio, también se condice con lo reportado en cifras intemacionales y destaca la importancia de diagnosticar a estos niños en esta etapa de la infancia, por el niesgo de ser hipertensos en el futuro y por lo tanto tener un mayor riesgo cardiovascular en la edad adulta. En un estudio de cohorte recientemente publicado, en que se estudiaron casi 15.000 niños y adolescentes, deja de manifiesto el subdiagnóstico de HTA y prehipertensión en la población pediátrica ${ }^{29}$. Los autores adjudicaron los resultados al poco conocimiento de los rangos de presión arterial normal en niños y al desconocimiento del equipo de salud de los valores previos de presión arterial en cada niño al momento de la evaluación.

Destaca en nuestros resultados el que no encontramos una asociación directa significativa entre los niños con presiones arteriales anormales y el grado de obesidad. Sólo uno de los 7 niños era obeso y se encontraba en rango de prehipertensión. Se ha descrito ampliamente en la literatura que la población pediátrica con mayor peso pre- 
sentaría HTA enmascarada, es decir, serían normotensos en tomas de presión aislada de consulta y resultarían ser hipertensos al realizarse una monitorización ambulatoria continua de la presión arterial de $24 \mathrm{~h}$. El pesquisar un niño con HTA enmascarada no es un dato menor, ya que Lurbe y cols ${ }^{30}$ han descrito una prevalencia de hasta casi $10 \%$ en población joven obesa, además de catalogarla como un precursor de HTA sostenida e hipertrofia ventricular izquierda $(\mathrm{HVI})^{31}$. En este sentido, sería importante realizar un monitoreo de presión arterial continua de $24 \mathrm{~h}$ a la población de riesgo.

En la población estudiada se encontraron cifras tensionales más elevadas en los hijos de padres hipertensos, lo cual apoya lo descrito previamente en la literatura en relación al antecedente familiar como factor de riesgo independiente de HTA ${ }^{16}$. El antecedente familiar de HTA es un factor de relevancia en nuestro estudio, ya que todos los niños hipertensos resultaron ser hijos de hipertensos, así como también uno de los prehipertensos. Cabe destacar que Robinson y $\operatorname{cols}^{32}$ al analizar una base de datos de 321 niños y adolescentes hipertensos demostró que el antecedente familiar de HTA era de gran peso encontrándose presente en 51\% y $26 \%$ de los hipertensos primarios y secundarios, respectivamente.

Estudios en adultos han mostrado que los niveles de PCRus se asocian a mayor riesgo cardiovascular e HTA. Datos similares han sido recientemente homologados en estudios pediátricos en donde ha quedado de manifiesto que una PCRus elevada está significativamente asociada en niños con obesidad ${ }^{33}$ e HTA esencial $^{34}$. En esta misma investigación se postula que la reducción de la presión arterial con bloqueadores de enzima convertidora e hidroclorotiazida disminuyen la hipertrofia ventricular con disminución de los niveles de PCRus. Assadi y cols ${ }^{35}$ continuando con su

\section{REFERENCIAS}

1. HajJar I, Kotchen T. Trends in Prevalence, Awareness, Treatment, and Control of Hypertension in the United States, 1988-2000. JAMA 2003; 290: 199-206.

2. Ingelfinger J. Pediatric Antecedents of Adult Cardiovascular Disease - Awareness and Intervention. N Engl J Med 2004; 350: 2123-6.

3. Marcun NV, Gregoric A. A diagnostic approach for the child with hypertension. Pediatr Nephrol 2005; 20: 499-506. línea de investigación, han publicado recientemente una asociación significativa entre PCRus e hipertrofia ventricular izquierda en niños y adolescentes con HTA esencial. Cabe destacar que datos en población adulta sugieren que la HTA es parte de un desorden inflamatorio al encontrar que niveles elevados de PCRus se han asociado con el desamollo de HTA futura $^{36}$. Se sabe que la PCRus disminuye la producción de óxido nítrico, lo cual puede promover la vasoconstricción. Así mismo, la PCRus tiene una acción proaterogénica, produciendo una regulación hacia amiba de la expresión del receptor de angiotensina 1. Nuestros resultados apoyan la relación entre HTA e inflamación, si bien no demuestran causalidad.

Encontramos que aquellos niños con PA anormal presentaron significativamente mayor nivel de PCRus, pero no observamos diferencias al analizar este parámetro según antecedente familiar, a diferencia de lo descrito en estudios recientes intemacionales en donde la descendencia de padres hipertensos presentan niveles significativamente mayores de PCRus ${ }^{37}$. La muestra de nuestro estudio, sin embargo, es muy pequeña, por lo cual se necesitan nuevos estudios sobre este tema, con poblaciones mayores para efectuar comparaciones.

En conclusión, la prevalencia de HTA encontrada en esta muestra es comparable a la reportada en la literatura. El antecedente de HTA en la familia se asocia a mayores cifras tensionales en los hijos. Los niños con presión arterial anormal tienen un mayor nivel de inflamación subclínica. Por tanto, creemos que la medición regular de la presión arterial, así como la identificación de factores de riesgo, permiten pesquisar a los niños hipertensos en forma precoz, quienes ya presentan evidencia de un estado proinflamatorio, asociado a mayor riesgo cardiovascular en la vida adulta.

4. Sorof JM, Lai D, Turner J, Poffenbarger T, Portman RJ. Overweight, ethnicity and the prevalence of hypertension in school-aged children. Pediatrics 2004; 113: 475-82.

5. Sorof JM, Poffenbarger T, Franco K, Bernard L, PoRTMAN RJ. Isolated systolic hypertension, obesity, and hyperkinetic hemodynamic states in children. J Pediatr 2002; 140: 660-6.

6. Muzzo S, Cordero J, Burrows R. Cambios en la prevalencia del exceso de peso del escolar chileno en los últimos ocho años. Rev Chil Nutr 1999; 26 : 311-5. 
7. Vio F, Albaia C. La transición nutricional en Chile. Rev Chil Nutr 1998; 25: 11-20.

8. Barja S, Arteaga A, Acosta AM, Hodgson Mi. Resistencia insulínica y otras expresiones del síndrome metabólico en niños obesos chilenos. Rev Méd Chile 2003; 131: 259-68.

9. Casanueva V, Cid X, Chiang MT, Román R, Milos C, Reyes M et al. Perfil lipídico y prevalencia de dislipidemias en niños y adolescentes de Concepción, Chile. Rev Méd Chile 1996; 124: 1453-61.

10. Encuesta Nacional de Salud 2003; Fundación de Cardiología de Chile y Bayer 2004.

11. BerRíos X. Tendencia temporal de los factores de riesgo de las enfermedades crónicas: ¿La antesala silenciosa de una epidemia que viene? Rev Méd Chile 1997; 125: 1405-7.

12. 2006. Estadística JUNAEB, 2006. www.junaeb.cl

13. Freedman DS, SRinivasan SR, Valdez RA, Wimamson DF, BERENSON GS. Secular increase in relative weight and adiposity among children over two decades: the Bogalusa Heart Study. Pediatrics 1997; 99: 420-6.

14. Arnaiz P, Acevedo M, Barja S, Berríos X, Guzmán B, BAmBS $C$ ET AL. Arterioesclerosis subclínica, factores de riesgo cardiovascular clásicos y emergentes en niños obesos chilenos. Rev Chil Pediatr 2007; 78: 137-44.

15. Blonde CV, Webier LS, Foster tA, Berenson GS. Parental history and cardiovascular disease risk factor variables in children. Prev Med 1981; 10: 2537.

16. SAIEH C. Factores de riesgo en hijos de padres hipertensos. Rev Med Clínica Las Condes 1995; 6: 15-7.

17. Van den Eizen AP, de Ridder MA, Grobbee DE, Witteman JC, UtiernaAl CS. Families and the natural history of blood pressure. J Hypertens 2004; 17: 936-40.

18. Marcun NV, GregorIC A. A diagnostic approach for the child with hypertension. Pediatr Nephrol 2005; 20: 499-506.

19. National Health and Nutrition Examination Survey. Anthropometry Procedures Manual. http// www.cdc.gov/nchs/data/nhanes/bm.pdf 2002.

20. MINSAL, Unidad de Nutrición: Norma para la evaluación nutricional del niño, entre 6 y 18 años. In. Santiago: MINSAL; 2003.

21. Fernández J, Redden D, Pietrobeu A, Auson D. Waist circumference percentiles in nationally representative samples of african-american, european-american an mexican-american children and adolescents. J Pediatr 2004; 145: 439-44.

22. Frisancho AR. New norms for upper limb fat and muscels areas for assesment of nutritional status. Am J Clin Nutr 1981; 34: 2540-5.

23. Slaughter MH, Lohman TG, Bolieau RA. Skinfold ecuation for estimation of body fatness in children and youth. Hum Biol 1998; 60: 709-23.
24. National High Blood Pressure Education Program Working Group on High Blood Pressure in Chidren and Adolescents. The Fourth report on the Diagnosis, Evaluation, and Treatment of High Blood Pressure in Children and Adolescents. Pediatrics 2004; 114: 55576.

25. Friedwald WT, LeVY RI, Frederickson DS. Estimation of plasma low density lipoprotein cholesterol concentration with use of preparative ultracentrifugation. Clin Chem 1972; 18: 499-509.

26. Sorof JM. Systolic hipertension in children: benign or beware? Pediatr Nephrol 2001; 16: 517-25.

27. Fuynn J. Differentiation Between Primary and Secondary Hypertension in Children Using Ambulatory Blood Pressure Monitoring. Paediatrics 2002; 110: 89-93.

28. Borges L, Peres M, Horta B. Prevalence of high blood pressure among schoolchildren in Cuiabá, Midwestern Brazil. Rev Saúde Pública 2007; 41: 530-8.

29. Hansen M, GunN P, Kaelber D. Underdiagnosis of Hypertension in Chidren and Adolescents. JAMA 2007; 298: 874-9.

30. Lurbe E, Invitti C, Torro I, Maronati A, Aguilar F, SARTORIO G ET AL. The impact of the degree of obesity on the discrepancies between office and ambulatory blood pressure values in youth. J Hypertens 2006; 24: $1557-64$.

31. Lurbe E, Torro I, Alvarez V, Nawrot T, Paya R, Redon J, Staessen J. Prevalence, Persistence, and Clinical Significance of Masked Hypertension in Youth. Hypertension 2005; 45: 493-8.

32. Robinson RF, Batisky DL, HaYes JR, Nahata MC, Mahan JD. Body mass index in primary and secondary pediatric hypertension. Pediatr Nephrol 2004; 19: 1379-84.

33. Acevedo M, Arnaiz P, Barja S, Bambs C, Berríos X, Guzmán B ET AL. Proteína $C$ reactiva y su relación con adiposidad, factores de riesgo cardiovascular y aterosclerosis subclínica en niños sanos. Rev Esp Cardiol 2007; 60: 1051-8.

34. Assadi F. C-reactive protein and incident left ventricular hypertrophy in essential hypertension. Pediatr Cardiol 2007; 28: 280-5.

35. AsSADI F. Relation of left ventricular hypertrophy to microalbuminuria and C-reactive protein in children and adolescents with essential hypertension. Pediatr Cardiol 2008; 29: 580-4.

36. Sesso H, Buring J, Rifai N, Blake G, Gaziano JM, Ridker P. C-reactive protein and the risk of developing hypertension. JAMA 2003; 290: 2945-51.

37. Díaz J, Arguelles J, Málaga I, Perilán C, Diéguez A, Vijande M, Málaga S. C-reactive protein is elevated in the offspring of parents with essential hypertension. Arch Dis Child 2007; 92: 304-8. 